\title{
HISTORIA DE LOS BLOGS
}

\section{José Francisco Durán Medina: Universidad de Castilla La Mancha (España)}

\section{Resumen}

El mundo de los blogs está entrando con fuerza en todos los ámbitos de la sociedad, incluso en los educativos, que están iniciando algunas investigaciones respecto a la utilización de esta novedosa herramienta, tan atractiva y motivadora para sus usuarios, cobrando especial interés pedagógico como herramienta de Érase una vez... intercomunicación entre profesor y alumnos.

Verdaderamente podríamos comenzar de esta manera ya que el tema tratado tiene un peculiar encanto parecido al de los cuentos. Además, para aumentar el interés, podemos añadirle una fuerte dosis de dificultad: su rabiosa actualidad, lo que hace prácticamente imposible encontrar documentación al respecto. Pero, si bien probablemente no podamos tildar aún de teorías la mayoría de textos hasta ahora escritos sobre este asunto, sí es cierto que comienzan a proliferar los mismos y que, en un futuro no muy lejano, casi con toda seguridad, las investigaciones venideras gozarán de un amplio repertorio para extraer información y poco a poco ir rellenando este vacío existente en el presente. No obstante, demostrando esta incipiente tarea recopilatoria, se expondrán a continuación algunos de los documentos más importantes sobre los blogs.

Comenzando por Lorenzo García Aretio, Titular del CUED (Cátedra UNESCO de Educación a Distancia) y Editor del BENED (Boletín Educación a Distancia), podríamos citar varios de sus artículos en dicha revista electrónica durante el año 2005 (junio y septiembre), donde aludiendo a las innegables ventajas que ofrece el fenómeno Internet en la formación y educación, y centrándose en esta nueva herramienta, el weblog, concluye afirmando y asegurando la existencia de numerosas bitácoras cuyo objetivo es la educación, amén de otras muchas dedicadas a conformar comunidades o grupos de trabajo colaborativo a nivel docente e investigador (García, 2005).

También en los Textos Universitaris de Biblioteconomía i Documentació de la Universitat de Barcelona (bid), Jorge Franganillo y Marcos Antonio Catalán nos describen las bitácoras y la sindicación de contenidos como utensilios para difundir información en bibliotecas y en los ámbitos de la educación y la investigación, definiendo claramente cada una de estas herramientas, haciendo una reseña histórica comentando los usos que de ellas se han hecho y planteando aplicaciones posibles en el entorno bibliotecario y en espacios de investigación (Franganillo y Catalán, 2005).

En la misma línea, intentando demostrar la aplicación de los weblogs en las bibliotecas, Dekialli Documental (2006) publica un artículo muy interesante en donde nos brinda enlaces a bibliotecas que están utilizando los blogs señalando que en el contexto hispanoamericano los biblioblogs pueden contarse con los dedos de la 
mano. Rebecca Blood, en su artículo Weblogs: una historia y una perspectiva (traducción propia), además de volver a recordarnos brevemente la historia de este recurso educativo, nos expone la perspectiva de futuro que proyecta, llegándolo a comparar con el antídoto que podrá resolver la saturación o bombardeo de datos que sufrimos sin la posibilidad de expresar nuestras propias ideas. "Blogeando cada día, el estudiante se hará un escritor con confianza en sí mismo. Leyendo lo que bloguean los demás compañeros evaluarán con interés lo que se dice. Leyendo y escribiendo pueden iniciar un viaje de autodescubrimiento intelectual" (Blood, R., 2000).

La Blogosfera Hispana: pioneros de la cultura digital, bajo la dirección de José M. Cerezo (2006) y con la aportación de importantes autores como José Cervera, Adolfo Estalella, Fernando Tricas, Juan J. Merelo, Víctor R. Ruiz, Gemma Ferreres, Fernando Garrido, José A. del Moral, Juan Varela, Juan Zafra, Ignacio Escolar, Rafael Chamorro, Fernando Polo, Enrique Dans, Hernán Casciari, J.A. Gelado, Vicent Partal o Antonio Fumero, tiene la clara vocación de ser una herramienta divulgativa de los beneficios de las nuevas tecnologías. $Y$ en este caso, se convierten en los autores del primer gran libro sobre la blogosfera hispana, en el que nos hablan del blog como fenómeno, como historia, como producto, como negocio, desarrollando la idea de los hiperenlaces como mecanismo sobre el que se elabora el concepto de conversación, criticándose, referenciándose, resumiendo lo dicho por el otro, etc.

El primer libro escrito y editado en castellano que trata exclusivamente y de forma multidisciplinar el fenómeno blog lleva el título: Blogs. La conversación en Internet que está revolucionando medios, empresas y ciudadanos y lo publica ESIC Editorial, dentro de su nueva Colección Divulgación. Los autores son todos miembros reconocidos de la blogosfera hispana, que han escrito sobre aquello en lo que son expertos: José Luis Orihuela (Weblogs y blogosfera), José Luis Antúnez (Impacto de los CMS en el despegue del fenómeno), Juan Varela (Periodismo participativo: El Periodismo 3.0), Julio Alonso (Blogs y empresas) y Octavio Rojas, que también hizo las veces de coordinador (Blogs y Relaciones Públicas). Como complemento a estos capítulos, los autores han entrevistado a varios personajes de la comunidad hispana e internacional, han redactado un glosario de términos habituales usados en la blogosfera y han reservado un capítulo práctico de publicación exclusiva en el blog (VV.AA., 2005).

Podíamos continuar citando gran cantidad de autores que, en los últimos años, han contribuido al conocimiento del mundo de los blogs mediante diversos artículos y ponencias (accesibles en http://mundoblog06.blogspot.com, 2006). Algunos ejemplos: José Luis Orihuela (Formato Blog y Cultura), Jaime Alonso (Comprender las Bitácoras desde el entramado mediático de Internet), Daniel Villar (Vidas compartidas con Imágenes), Adriano Morán (Jabalí Digital), Inmaculada Bermejo (Chavalina.net), Juan Carlos García (La Usabilidad de las Bitácoras como factor de éxito), Diego Sevilla (Planet Murcia, Sindicación y Weblogs desde la Universidad de Murcia), Julio Alonso (Nanomedios: los Blogs como medio online y como negocio), Juan Luis Hortelano y Sergi Torres (Blog y Empresa: ¿para todos?).

En cuanto al campo educativo que nos interesa, la revista electrónica Edutec-L, igualmente se ocupa de vez en cuando de este fenómeno novedoso. El número de 
julio/agosto de 2003 The Technology Source incluye varios artículos sobre la utilización y el impacto de los weblogs (diarios personales, cuadernos de bitácora) en educación; comenta que Stephen Downes en Weblogs at Harvard Law, partiendo de una experiencia pionera, dice que cualquier miembro de Harvard puede disponer de su propio weblog; Mary Harrsch (2003), en RSS: The Next Killer App for Education expone el potencial de la sindicación de contenidos en educación; y Jon Baggaley, en Blogging as a Course Management Too" refiere el uso de weblogs como herramienta para publicar materiales educativos.

Del mismo modo, la revista DIM (Didáctica, Innovación y Multimedia), en marzo del año 2006, razona su teoría sobre el uso de las bitácoras como herramienta de optimización del aprendizaje, comentando que:

"Las Nuevas Tecnologías de la Información y Comunicación (TIC) nos sitúan en un momento en el que el alumno tiene una mayor facilidad de acceso a una cantidad muy superior de información, por lo que el reto consiste básicamente en desechar una sobrecarga de contenidos que, por otro lado, pueden encontrar en la red con suma facilidad, para enseñar a aprender, y fomentar una actitud crítica ante lo que lee, escucha y ve en los medios de comunicación de masas, a través del análisis de noticias aparecidas en medios de comunicación y relacionadas con los temas que componen la parte teórica de la asignatura. Es decir, que la parte práctica de la asignatura respalde en la medida de lo posible, los contenidos teóricos de la asignatura, y a la vez facilitar un espacio de debate doblemente público, por un lado en el aula, y por otro en la misma Red a través de los weblogs, accesibles por todos los internautas, que pueden dejar sus aportaciones a través de comentarios en cada entrada" (Blanco, 2006).

La misma revista, en el año 2005, publicó el artículo Aprendiendo a expresarse con Weblogs (Robles, 2005), donde decía que "uno de los problemas fundamentales de nuestros estudiantes es enseñarles a escribir. No sólo tienen el problema de cometer faltas de ortografía, sino que además tienen dificultad para saber expresar lo que quieren decir por escrito. Iniciativas como hacerles escribir redacciones o hacer un periódico escolar son buenas, pero un weblog tiene un efecto motivador grande, que hace que el estudiante se esfuerce al máximo ya que está expuesto a la crítica de todo el mundo (compañeros, familiares, profesores y desconocidos) y no sólo a la de su profesor de turno" (http://www.pangea.org).

Glen Bull, Gina Bull y Sara Kajder (2004) escriben Writing with Weblogs, publicado en el Número 1 del Volumen 31 de la revista Learning \& Leading with Technology, y traducido por Eduteka en junio del año 2004 (accesible en http://www.eduteka.org), donde describen la escritura con weblogs como una increíble e interesante oportunidad para los diarios estudiantiles, dado que su estructura crea un marco para las interacciones sociales y activa el deseo básico de las personas para interconectarse y comunicarse, asegurando la gran capacidad que tiene para impartir instrucción.

En el número 2 del mismo Volumen 31, aparece un artículo de Bull y Kajder (2003) titulado Scaffolding for Struggling Students en el que aseguran que la lectura y 
escritura con blogs sirven de apoyo para estudiantes con dificultades en estas áreas, además de poder convertirse en mucho más que un diario en línea y tener diversas aplicaciones que pueden usarse para enseñar).

Entre algunos de los artículos y ponencias de Eland Vera debemos destacar Los Weblogs como Herramienta Educomunicativa, donde se nos expone que la sociedad de la información y el conocimiento ha permitido la emergencia del fenómeno blog o página web personal, su multiplicación es constante y ya comienzan a surgir profesores que la emplean como herramienta educomunicativa, sus ventajas son analizadas y se recomienda la alfabetización digital a fin de acceder a la creación y mantenimiento de edublogs, pues permiten la construcción del conocimiento y la exteriorización de la identidad del alumno.

\section{Vera escribe:}

"Este apoyo a diferencia de otros recursos educativos tiene la singularidad de hacer realidad el ansiado sueño de la pedagogía constructivista: permitir la construcción o hechura del conocimiento teniendo como eje al aprendiz, pues el propio estudiante junto al maestro es autor de su propio blog. La función del profesor, visto de esta manera, es la de mediador y guía, haciendo realidad otro ansiado sueño, el de la teoría del aprendizaje social o aprendizaje por observación de modelos: el estudiante navega en la blogosfera educativa procesando las pautas, recomendaciones, alertas e inconvenientes del maestro y de sus compañeros" (Vera, 2006).

Gorka Palacio (2006) en Weblearner.Info nos explica cómo poco a poco, las universidades de todo el mundo irán incorporando las granjas de bitácoras o edublogs (blogs para la Educación) entre sus servicios para la comunidad estudiantil. Comenta que los primeros ejemplos ya se están viendo en los países desarrollados, como la Universidad de Waterloo en Ontario (Canadá), que ya ha puesto en modo de prueba lo que parece va a ser una gran red de weblogs para los estudiantes de su universidad.

José Luis Orihuela, en La revolución de los blogs, dando ya por sentada la importancia de aplicar las nuevas tecnologías en educación, define con gran claridad las ventajas que nos puede ofrecer la utilización de blogs en las aulas. Dada su importancia, se estima conveniente su transcripción literal:

"Existen tres ventajas básicas de los weblogs frente a las páginas web convencionales por las que se recomienda su adopción en el ámbito educativo:

- El manejo de herramientas para la creación y publicación de weblogs es más sencillo, y por tanto su aprendizaje más breve, respecto del proceso de edición de páginas web en editores HTML y publicación en servidores web mediante FTP.

- El estilo visual de los weblogs, basado en plantillas predefinidas, resuelve el diseño gráfico, permitiendo a los alumnos centrarse en los contenidos y en el proceso de comunicación.

- Los weblogs ofrecen una serie de funciones integradas como los comentarios, la fecha y hora de publicación, la detección automática de referencias (trackback), el sistema de archivos, los buscadores internos 
y los enlaces permanentes individuales de las historias publicadas, que aportan valor agregado a la producción de contenidos en línea.

Los weblogs ayudan a los alumnos a convertirse en expertos, aumentan el interés de los estudiantes en el aprendizaje y abren cauces efectivos de participación que facilitan el diálogo y el discurso académico" (Orihuela, 2006).

No podemos olvidarnos de la existencia de algunos detractores de los mismos quienes, aunque pocos, también existen, sobre todo si, como dice Juan Varela (accesible en http://www.librodeblogs.com), asimilan los blogs con un medio de comunicación social y les exigen las garantías del periodismo olvidando que también son, sobre todo, un medio de comunicación interpersonal, como la charla. Éste es el caso de José María Pozuelo Yvancos, quien afirmó que aborrecía de la forma y sentido que han adoptado algunos de los llamados blogs, o espacios de comunicación abierta en Internet (Varela, 2005).

También queremos reseñar aquí un original y atrevido artículo en NoticiasDot.com, el día 19 de septiembre de 2005, titulado Escribir en un Blog reemplaza al Psicólogo y asegura un efecto terapéutico del mismo según el cincuenta por ciento de los seiscientos bloggers encuestados por American Online.

De acuerdo con la teoría de Jorge Franganillo y Marcos Antonio Catalán (Franganillo y Catalán, 2005), la primera página web ya era un weblog, por lo que tendríamos que remontarnos a 1989 para encontrar el origen de esta herramienta, cuando el científico británico Tim Berners-Lee, el padre de Internet, diseñó una página incorporando múltiples enlaces a sitios web de nueva aparición.

No obstante, la acuñación del término propiamente dicho, así como sus derivaciones y abreviaturas, se debe a Jorn Barger, Padre del Weblog, quien en 1997 presentó su famoso The Robot Wisdom Pages, página dedicada casi en su totalidad a ofrecer enlaces interesantes, donde aparece por primera vez esta palabra compuesta por Web y Log (registro, cuaderno de bitácora). Dos años más tarde, Peter Merholtz (1999) en su página Peterme (accesible en http://www.peterme.com), separó este término en We Blog, dando lugar a la abreviatura conocida.

A partir de entonces, estos vocablos son adoptados por la gran mayoría de usuarios $y$, de una manera sorprendentemente creciente, van apareciendo multitud de weblogs en los años sucesivos. Veamos los aspectos más relevantes cronológicamente:

- Año 1999. El fenómeno del weblog y de las páginas web actualizadas periódicamente llega a los países de habla hispana: Bitácora Tremendo, del mexicano Carlos Tirado (accesible en http://tremendo.com), Área Estratégica, del mexicano Gustavo Arizpe (accesible en http://www.areaestrategica.com), $\cdots$

- Año 2000. Aparece el término blogosfera como red de blogs interconectados. También hacen su aparición los primeros blogs de autores españoles. Aparece Earcos.blogspot.com del ecuatoriano Eduardo Arcos (estuvo accesible en http://earcos.blogspot.com, actualmente en 
http://eduardo.arcos.cc/), quien poco más tarde crea el primer directorio de blogs, bitacoras.net. Luciano A. Ferrer escribe el primer mensaje abierto al público en la bitácora ya desaparecida de la revista MeArte. Alberto González (Beto) visita los laboratorios Pyra Labs, creadores de Blogger. El día trece de abril, Olalla Cernuda publica un curioso artículo con el título Enlazo, luego existo (accesible en http://www.baquia.com). Javier Cañada crea Terremoto.net, primer blog dedicado a tratar la usabilidad en lengua española (accesible en http://www.terremoto.net). En octubre aparece Yonkis.com, posiblemente el primer weblog no autorizado para menores (accesible en http://www.yonkis.com).

- Año 2001. Los weblogs se extienden con rapidez asombrosa. Antonio Cambronero escribe su primera anotación en Blogpocket (accesible en http://www.blogpocket.com). Ramón Buenaventura escribe en su web: "Este añadido a mi página web es lo que se llama un 'weblog', un cuaderno de bitácora de Internet. Se trata de una técnica relativamente nueva. Dentro de un tiempo aparecerá en El semanal un artículo mío sobre el tema. Mientras tanto, quienes lean inglés pueden ponerse al día en Blogger. Anímense y creen su propio cuaderno" (http://www.rbuenaventura.com). A finales de enero Beto González, con Pura Vida (accesible en http://www.betobeto.com), consigue el Premio al Mejor Blog latinoamericano en un Certamen Internacional dedicado a los weblogs. En marzo, Diego Martín Lafuente estrena uno de los primeros weblogs de la blogosfera argentina: Minid.net (estuvo accesible en http://www.minid.net, actualmente no). Aparece Marianitu, famosa bitácora de Miguel Ángel Esteban (accesible en http://www.marianitu.net). En agosto, Joaquín Bernal en Earful expone un truco para adaptar al español los formatos de fechas en los blogs de blogger.com (estuvo accesible en http://earful.bitako.com, también inaccesible en la actualidad). También en este año se da a conocer Greenbloggers, comunidad de importantes autores como Kapa, Inetd, Zor, Ichabod... cerrada en el año siguiente (2002). El seis de diciembre, Mercé Molist escribe que los diarios de navegación triunfan en Internet, entre otras cosas, por la frescura de sus contenidos (http://ww2.grn.es). La Hormiga Remolona de Rosana Ferreres (accesible en http://www.hormigaremolona.com), Log de Literatura, gana el Premio a la Mejor Bitácora de Arte y Cultura. También en diciembre, aunque su verdadera creación fuera el $\mathbf{3 0}$ de noviembre, aparece en Tira Ecol (accesible en http://www.tiraecol.net/) una tira cómica de Bilo y Nano señalando la importancia social de los blogs.

- Año 2002. Se producen los primeros encuentros presenciales de los autores de los blogs; comienzan igualmente a proliferar los premios específicos de los weblog; y los profesores de universidades se empiezan a interesar por este fenómeno. En enero se reúnen en Barcelona blogueadores tan conocidos como Mia Font (2001, 4Colors: accesible en http://www.4colors.net), Miguel Ángel Esteban, Alex Grau (Webugger, actualmente dominio de otra persona), Fernando Caballero (Bitácora de Mierda y Divinas Palabras: http://divinaspalabras.blogspot.com). Nace Blogalia, herramienta sencilla editora de blogs (accesible en http://www.blogalia.com). Primer relato de un viaje realizado por un estudiante (Marín, I., 2002) en español (accesible en 
http://web.archive.org). El 2 de marzo de este mismo año, surge la primera reunión de bitacoreros españoles en Madrid: (estuvo accesible en http://www.nocompares.com/kedada). También en marzo se conceden los Premios Webloggeros y Eso (igualmente estuvo accesible en http://www.bulletrain.net/premios), primeros en España. En abril, más concentraciones, esta vez en México, con los famosos Carlos Tirado, Gustavo Arizpe y Eduardo Arcos. En junio nace el Blogómetro (accesible en http://blogometro.blogalia.com), en julio el periodista Juan Varela crea Periodistas 21 (accesible en http://www.periodistas21.com) y en agosto, además de la inauguración de la weblog del profesor universitario de Navarra José Luis Orihuela eCuaderno (accesible en http://www.ecuaderno.com), nos llama particularmente la atención la aparición de los Antipremios, porque tú lo vales como reacción al buen ambiente general en la blogosfera (estuvo accesible en http://www.antipremios.es.vg). El Sentido de la Vida, blog dedicado a los Monty Python, gana el Premio 20Blogs por votación popular (premios accesibles en http://www.20minutos.es). El nueve de diciembre comienza el lanzamiento de la primera campaña protesta mediante blog Prestige: exigimos responsabilidades (anteriormente estuvo accesible en http://www.librodenotas.com/mt/prestige.html), encontrando gran aceptación y seguimiento entre los usuarios.

- Año 2003. Aunque realmente se crea en 2001, es en 2003 cuando se estabiliza y da a conocer Dialógica (accesible en http://www.dialogica.com.ar), conjunto de blogs sobre educación, periodismo, política, nuevas tecnologías y comunicación. El diario argentino Clarín se convierte en el primer medio de comunicación de masas de habla hispana que incluye un blog creado en 2001 por Mariano Amartino (accesible en http://www.uberbin.net). En abril, Microsiervos.com publica un mapa sobre la blogosfera hispana (accesible en http://www.microsiervos.com). Entre la gran cantidad de blogs nuevos podemos destacar el del profesor de Área de Sistemas y Tecnologías de la Información del Instituto de la Empresa, Enrique Dans (en la actualidad accesible en http://www.enriquedans.com), y también Error500, blog sobre las Tecnologías de la Información, escrito en principio por varios autores y finalmente editado sólo por Antonio Ortiz (accesible en http://www.error500.net). Otros blogs importantes son Escolar.net, de Ignacio Escolar (accesible en http://www.escolar.net), Guaiquil.net, de Rodrigo Guaiquil (estuvo accesible en http://www.guaiquil.net, página web redireccionada actualmente a http://mediosdigitales.info/), Diari de Campanya, de Miquel Iceta (accesible en http://www.iceta.org). Y siguen proliferándose las reuniones, como el I Encuentro Físico de Bloggers, Ges, Freaks, Internautas, Hackers y demás 'fauna' de la red en Aragón, Beers \& Blogs primero en Pamplona (accesible en el blog de Orihuela, J.L.: http://blogzine.blogalia.com) y luego en A Coruña y Madrid (igualmente accesible en http://blogzine.blogalia.com). En septiembre aparece la primera blogonovela en español cuyo título es Weblog de una mujer gorda, inicialmente publicado en Blogger.com y posteriormente publicado en Bitacoras.com con el título Más respeto, que soy tu madre (Casciari, H., accesible en http://mujergorda.bitacoras.com). Y en octubre se da a conocer Bitacoras.com, que junto a blogger y blogia forman las más importantes herramientas de diseño y edición de blogs (accesible en 
http://www.bitacoras.com). A finales de año es cuando comienza a aparecer publicidad en los blogs españoles, habitual tema de conversación en los mismos durante un periodo considerable de tiempo. También en este mes tiene lugar el nacimiento de Filmica.com, comunidad de blogs temáticos (accesible en http://www.filmica.com).

- Año 2004. Siguen aumentando tanto las reuniones presenciales como la creación de nuevos blogs. Aparece el primer programa televisivo dedicado a los blogs titulado Internet@mano, emitido por Localia TV. Aparece un ranking con los cien blogs más visitados según Technorati (accesible en el blog de Juan Julián Merelo: http://atalaya.blogalia.com). Surgen nuevas convocatorias, como Tacos y Blogs, primer encuentro de bloggers mexicanos con la asistencia de 8 personas, o la de Cervezas y Blogs, con un mes de separación y más de cien personas, o el Primer Pizzas y Blogs en Sevilla. En este año, durante el mes de abril, tiene lugar la creación de Blogs.ya.com, creador y editor de weblogs introducido en un espacio web muy conocido (accesible en http://blogs.ya.com). Siguen apareciendo weblogs de interés, como Atinachile en Chile (accesible en http://www.atinachile.cl), La Red Opina creado por Wanadoo en su espacio sobre actualidad, actualmente Orange (accesible en http://actualidad.orange.es), Periodismo Global creado por Fernando Meza (accesible en http://periodismoglobal.blogspot.com), Xataka (blog de Sacha Fuentes, Javier Penalva y Whiskito: accesible en http://www.xataka.com) dedicado a los gadgets y la electrónica de consumo, etc. En agosto de este año, Miguel Ángel Esteban imparte un taller de weblogs denominado Campusmac. El seis de diciembre se otorgan los Premios BOBs (Best Of the Blogs), resultando ganadores los blogs en español El hombre que comía diccionarios de Javier Arce recibiendo el premio como mejor tema (accesible en http://www.elhombrequecomiadiccionarios.com), La Malarosa de Moon-tse como mejor diseño (creado el 31 de diciembre del año 2003 y accesible en http://www.lamalarosa.com), Periodistas21 de J. Varela como mejor blog periodístico (http://periodistas21.blogspot.com) y Caspa.tv de Antonio Delgado (creado en 2001 y accesible en http://www.caspa.tv) recibió el premio del público. $\mathrm{Y}$ el año termina con un espectacular Blogomaratón Solidario el día 21 de diciembre, donde por espacio de 24 horas gran cantidad de blogs se unieron con el fin de presentar los proyectos de distintas ONGs y las maneras de colaborar con ellas (blogomaratones anualmente accesibles hasta el 2005 en http://blogomaraton.blogia.com).

- Año 2005. Continúan convocando premios y realizando listas con el ranking de los blogs más visitados, como Los 100 blogs en español de Atalaya también según Technorati como en anteriores ocasiones (blog de J. J. Merelo, accesible en http://atalaya.blogalia.com), Premios 20Blogs del diario 20minutos (accesible en http://www.20minutos.es), Premios Bitácoras.com 2004 otorgando dieciocho premios y dejando uno desierto (premios accesibles en http://www.bitacoras.com), etc. Los blogs irrumpen en los medios de comunicación de masas: Qué!, diario gratuito a partir de blogs creados por los lectores (estuvo accesible en http://www.quediario.com, actualmente en http://www.que.es/), Diario Vasco abre una sección dedicada a los blogs de los lectores (accesible en http://blogs.diariovasco.com), Lacuarta (accesible en http://blog.lacuarta.cl) blog del diario chileno La 
Cuarta, Dvinosblog del diario El Mercurio de Santiago de Chile (estuvo accesible en http://www.dvinosblog.cl), El Correo Digital de Bilbao con su sección de blogs (estuvo accesible en http://blogs.vizcaya.elcorreodigital.com, encontrándose actualmente en http://blogs.elcorreodigital.com/), etc. Se publica el primer libro en español cuyo título es Mi Vida Perra, que recoge los artículos del blog Antes Muerta Que Sencilla (estuvo accesible en http://www.lacoctelera.com/amqs, actualmente http://amqs.lacoctelera.net/). Algunos blogs importantes que aparecen durante este año son Recorramos juntos de Camilo Herrera (accesible en http://www.camiloherrera.cl), del mismo autor junto a otros compañeros es el metablog pedagógico Educándonos (accesible en http://www.educandonos.cl), un blog corporativo llamado eTc: El Blog de marketing en español (accesible en http://etc.territoriocreativo.es), Blog de PCActual (estuvo accesible en http://www.pc-actual.com), el blog sobre tecnología de consumo en español Engadget (accesible en http://es.engadget.com), Valenciablog con información turística de la ciudad (accesible en http://www.valenciablog.com), El Blog de John Mackenzie blog bilingüe en Santiago de Chile de gran interés para todos los que somos amantes de los temas educativos (accesible en http://jotamac.typepad.com), otro blog en español sobre gadgets con el nombre de Gizmodo (accesible en http://es.gizmodo.com), Blogalaxia directorio de blogs latinos (accesible en http://www.blogalaxia.com). También a lo largo del año 2005 comienzan a celebrarse mesas redondas acerca de este tema, con títulos como Los Weblogs en el periodismo, Los Blogs: valor estratégico y evolución previsible en el futuro, 1CWCOM, Primera Conferencia Weblogs-Comunicación en México, Blogs y Empresas y Mesa Redonda: La Empresa ante los blogs en Madrid, etc. La fuerza de los weblogs es tal que los propios partidos políticos hacen uso de ellos en sus campañas electorales: la candidata del Partido Popular a las elecciones autonómicas del País Vasco María San Gil (no existente en la actualidad, pero antes accesible en www.msangil.com), Blog de Campaña del candidato del Partido Nacionalista Vasco Juan José Ibarretxe (estuvo accesible en http://www.ibarretxe.com), el senador chileno Fernando Flores también publicó uno titulado Abriendo Juego, Abriendo Mundos (estuvo accesible en http://www.fernandoflores.cl)... Una curiosa novedad: Alberto Álvarez-Perea efectúa la retransmisión de una Semana Santa por medio de un blog (estuvo accesible en http://www.moonshadow.es). Otros acontecimientos de gran interés fueron el II Blogomaratón Solidario (convocatoria accesible en http://blogomaraton.blogia.com), la II Encuesta a webloggers y lectores de blogs (actualmente no disponible pero sí accesible la encuesta del año 2004 en http://www.blogpocket.com), también tiene lugar el primer Festival de Blogs bajo el epígrafe Bitácoras y Libertad de Expresión (accesible en el blog de Manuel Almeida: http://mangasverdes.es), Beers \& Blogs en Madrid (reunión mensual, en la actualidad accesible en el blog de Octavio Rojas: http://octaviorojas.blogspot.com), Día de Internet (estuvo accesible en http://www.diadeinternet.es) con la entrega del Premio al mejor Weblogger para Fernando Jáuregui, Primer Taller Legal para Bloggers organizado por la ONG Derechos Digitales en Santiago de Chile (actualmente accesible en http://www.derechosdigitales.org). Un dato importante es que Terra.com lanza 
su servicio de blogs (accesible en http://blog.terra.com). Y otro dato curioso: el Diccionario Panhispánico de Dudas recoge los términos Blog, Bitácora y Weblog (accesible en http://buscon.rae.es).

- Año 2006. Sería demasiado extenso citar todos los acontecimientos habidos en ese año con respecto a los blogs dado su crecimiento y expansión. Se han incorporado a la vida cotidiana de tal manera que únicamente serán citados una mínima cantidad de los relacionados con la Educación: múltiples ponencias (José Luis Orihuela, Jaime Alonso, Daniel Villar, Adriano Morán, Inmaculada Bermejo, Juan Carlos García, Diego Sevilla, Julio Alonso, Juan Luis Hortelano, Sergi Torres...), Deakialli publica un artículo muy interesante en donde nos brinda enlaces a bibliotecas que están utilizando los blogs (comenzando ya a utilizar términos nuevos como biblioblogs, accesible en http://deakialli.bitacoras.com). Proliferan gran número de noticias, como Ciencia en blog (accesible en http://portal.educ.ar) escrito por Pablo Mancini, De la imprenta al CMS de blogs por José Luis Antúnez (accesible en http://www.librodeblogs.com), State of the blogosphere de David Sifry (accesible en http://www.sifry.com), Y los blogs se hicieron visibles escrito por Juan Varela, (accesible en http://www.librodeblogs.com), y el Gobierno de Chile escribe Ciudadanía podrá enviar sus opiniones al Consejo Asesor Presidencial para la Calidad de la Educación a través de un blog (estuvo accesible en http://www.gobiernodechile.cl/noticias/detalle.asp), Weblog, un concepto romántico de la formación por Carlos Castaño Garrido (estuvo accesible en http://weblearner.info/?p=72), o el artículo ya mencionado Los weblog como herramienta educomunicativa de Eland Vera (accesible en http://autocosmofilia2.blogspot.com). Además, surgen ya diversas experiencias educativas en las aulas mediante la utilización de blogs, como las de Felipe Zayas con Gramática y Literatura para alumnos de la E.S.O. (accesible en http://felipezayas.googlepages.com) o sobre el Quijote (2005, accesible en http://leyendoelquijote.bitacoras.com) y de Lengua en general (accesible en http://darlealalengua.blogspot.com), los edublog en la Universidad de los que nos habla el catedrático en Tecnología de la Información Gorka Palacio (estuvo accesible en http://www.alianzo.com/resources/blogak/palazio_edublogak.pdf), el Superblog de Seminario elaborado por Mario Núñez donde aparece el término blogfesor (estuvo accesible en http://www.vidadigital.net/tecnociso), incluso el mío propio con alumnos de Tercero de Magisterio y seguramente futuros blogfesores (2005, accesible en http://blog-nntt.blogspot.com).

Como hemos podido comprobar, la vida de los edublogs, aunque todavía corta, claramente nos muestra su gran potencial educativo y ofrece ya un amplio repertorio de ejemplos que enmarcan todos los ámbitos de la sociedad incluyendo, cómo no, el aspecto educativo, tan necesitado de la motivación e interés que despierta esta nueva herramienta al servicio de todos los miembros de la Comunidad Educativa. 


\section{Referencias}

BARGER, J. (1997). The Robot Wisdom Pages [En Línea]. Blogger.Com 1997. Disponible En Web:

Http://Robotwisdom2.Blogspot.Com/

BLANCO, S. (2006). El Uso De Las Bitácoras Como Herramienta De Optimización Del Aprendizaje [En Línea]. Pangea.Org. Marzo De 2006. Disponible En Web:

\section{Http://Www.Pangea.Org/Dim/Revista4.Htm}

BLOOD, R. (2003). Diez Consejos Para Una Bitácora Mejor [En Línea]. Rebecca's Pocket (Versión Española De José Luis Orihuela). 22 Marzo 2003. Disponible En Web:

\section{Http://Www.Rebeccablood.Net/Essays/Ten Tips Espanol.Html}

BULI, G.; BULL, G Y KAJDER, S. (2004). Writing With Weblogs [En Línea]. Eduteka.Org. 5 Junio 2004. Disponible En Web:

Http://Www.Eduteka.Org/Weblogs1.Php

BULL, G Y KAJDER, S. (2003). Scaffolding For Struggling Students [En Línea]. Iste (International Society For Technology In Education). Octubre 2003. Disponible En Web: Http://Www.Iste.Org/Content/Navigationmenu/Publications/LI/Llissues/Volume 3120032004 /October3/October 2003. Htm

CEREZO, J. M. (Dir) (2006). La Blogosfera Hispana: Pioneros De La Cultura Digital [En Línea]. Fundación France Telecom España. 24 Abril 2006. Disponible En Web:

Http:/Www.Fundacionorange.Es/Areas/25 Publicaciones/La Blogosfera Hispa $\underline{\text { na.Pdf }}$

DEAKIALLI (2006). Bibliotecas Que Bloguean [En Línea]. Deakialli Documental.Com. 20 Marzo 2006. Disponible En Web:

\section{Http://Www.Deakialli.Com/2006/03/20/Bibliotecas-Que-Bloguean/}

FRANGANILLO, J. Y Catalán, M. A. (2005). Bitácoras Y Sindicación De Contenidos: Dos Herramientas Para Difundir Información [En Línea]. Bid Facultat De Biblioteconomia I Documentació Universitat De Barcelona. Diciembre De 2005. Disponible En Web: Http://Www2.Ub.Edu/Bid/Consulta Articulos.Php?Fichero=15frang2.Htm

GARCÍA ARETIO, L. (2005). Weblog - Bitácora [En Línea]. Editorial Del Bened. Junio De 2005. Disponible En Web:

\section{Http://Www.Uned.Es/Catedraunesco-Ead/Editorial/P7-6-2005.Pdf}

HARRSCH, M. (2003). Rss: The Next Killer App For Education [En Línea]. University Of North Carolina. Julio - Agosto 2003. Disponible En Web: 


\section{Http://Technologysource.Org/Article/Rss/}

MERHOLTZ, P. (1999). Peterme [En Línea]. Peterme.Com. Disponible En Web: Http://Www.Peterme.Com/

Mundoblog (2006). Primera Jornadas Regionales Sobre E-Culture [En Línea]. Blogger.Com. 1 - 3 Marzo 2006. Disponible En Web:

Http://Mundoblog06.Blogspot.Com/

Noticiasdot.Com (2005). Escribir En Un Blog Reemplaza Al Psicólogo [En Línea]. Noticias Digitales, S.L. 19 Septiembre 2005. Disponible En Web:

Http:/Www.Noticiasdot.Com/Publicaciones/2005/0905/1909/Noticias/Noticias 1 90905-10.Htm

ORIHUELA, J.L. (2006). La Revolución De Los Blogs. Madrid: La Esfera De Los Libros.

PALACIO, G. (2006). Los Edublogs En La Universidad [En Línea]. Blogak 2.0. 6 Abril 2006. Disponible En Web: Http:/Www.Bak.Ehu.Es/P255Content/Es/Contenidos/Informacion/Cursosverano Prog Sociedad/Es Socieda d/Adjuntos/070716 Gp.Pdf

ROBLES, R. (2005). Aprendiendo A Expresarse Con Weblogs [En Línea]. Pangea.Org. Febrero De 2005. Disponible En Web: Http://Www.Pangea.Org/Dim/Revista0.Htm

VARELA, J. (2005). Prejuicios De Mirar Los Blogs Desde La Lejanía [En Línea]. Librodeblogs.Com. 29 Diciembre 2005. Disponible En Web: Http://Www.Librodeblogs.Com/Caps/3/Prejuicios-De-Mirar-Los-Blogs-DesdeLa-Lejanaa.Php

VERA, E. (2006). Los Weblogs Como Herramienta Educomunicativa [En Línea]. Blogger.Com. 2 Febrero 2006. Disponible En Web: Http://Autocosmofilia2.Blogspot.Com/2006/02/Los-Weblogs-ComoHerramienta.Html

Vv.Aa. (2005). Blogs: La Conversación En Internet Que Está Revolucionando Medios, Empresas Y A Ciudadanos. Madrid: Esic. 